\title{
Angular momentum transport and turbulence in laboratory models of Keplerian flows
}

\author{
M. S. Paoletti ${ }^{1}$, D. P. M. van Gils², B. Dubrulle ${ }^{3}$, Chao $\operatorname{Sun}^{2}$, Detlef Lohse ${ }^{2}$, and D. P. Lathrop ${ }^{4}$ \\ 1 Department of Physics and Center for Nonlinear Dynamics, University of Texas, Austin, TX 78712, USA \\ e-mail: mpaolett@gmail.com \\ 2 Physics of Fluids Group, Faculty of Science and Technology, Mesa+ Institute and Burgers Center for Fluid Dynamics, University \\ of Twente, 7500AE Enschede, The Netherlands \\ 3 CNRS URA 2464 SPHYNX/SPEC/IRAMIS/DSM, CEA Saclay, 91191 Gif-sur-Yvette, France \\ 4 Departments of Physics and Geology, Institute for Research in Electronics and Applied Physics, Institute for Physical Science and \\ Technology, University of Maryland, College Park, MD 20742, USA \\ e-mail: lathrop@umd.edu
}

Received 23 November 2011 / Accepted 5 September 2012

\begin{abstract}
We present angular momentum transport (torque) measurements in two recent experimental studies of the turbulent flow between independently rotating cylinders. In addition to these studies, we reanalyze prior torque measurements to expand the range of control parameters for the experimental Taylor-Couette flows. We find that the torque may be described as a product of functions that depend only on the Reynolds number, which describes the turbulent driving intensity, and the rotation number, which characterizes the effects of global rotation. For a given Reynolds number, the global angular momentum transport for Keplerian-like flow profiles is approximately $14 \%$ of the maximum achievable transport rate. We estimate that this level of transport would produce an accretion rate of $\dot{M} / \dot{M}_{0} \sim 10^{-3}$ in astrophysical disks. We argue that this level of transport from hydrodynamics alone could be significant. We also discuss the possible role of finite-size effects in triggering or sustaining turbulence in our laboratory experiments.
\end{abstract}

Key words. accretion, accretion disks - turbulence - methods: laboratory - hydrodynamics

\section{Introduction}

Astrophysical disks are ubiquitous in the universe, orbiting around a wide panel of massive objects, from black holes to stars. An essential ingredient of their dynamics is the radial transport of angular momentum that governs the rate of material falling onto the central object. Realistic models of this process are impeded by the complexity of the disk dynamics, including a wide variety of processes such as general relativistic effects, selfgravitation, radiation, dynamics of plasma magnetic fields and turbulence. In particular, the contribution from hydrodynamics to the angular momentum transport in such astrophysical flows is not yet understood.

A natural first-step in the construction of a robust model is to consider the minimal list of ingredients that can capture and reproduce the observed disk properties. Such a model has been proposed several decades ago by Shakura \& Sunyaev (1973) and Pringle (1981), and was given the name $\alpha$ disks. In that model, the radial transport of angular momentum is parameterized by a turbulent viscosity coefficient that needs to be prescribed or measured. In the past, there have been several attempts to estimate this coefficient from an analytical closure model (Dubrulle 1992), numerical simulations (see e.g. Rozyczka \& Spruit 1993; Brandenburg et al. 1995; Abramowicz et al. 1996; Stone et al. 1996; Drecker et al. 1998; Beckwith et al. 2011; and a recent review by King et al. 2007, and references therein), or using theoretical arguments (Canuto et al. 1984; Mukhopadhyay 2008).

An alternative promising option, as recognized by Zeldovich (1981) and Richard \& Zahn (1999), is offered by instead focusing on laboratory experiments. Indeed, it may be shown
(Hersant et al. 2005) that under simple but well founded approximations the equations governing an $\alpha$ disk are similar to the equation of motion of an incompressible rotating shear flow, with penetrable boundary conditions and cylindrical geometry. This can be modeled in the Taylor-Couette flow (see Fig. 1), a fluid layer sheared between two independently rotating, coaxial cylinders.

These proposals motivated several studies of the transport properties in rotating shear flows, with contradicting conclusions. From the numerical point of view, the problem has been mainly tackled in the astrophysical community using the socalled shearing sheet approximation either without explicit viscosity (Balbus et al. 1996; Hawley et al. 1999), or with explicit viscosity (Lesur \& Longaretti 2005) at resolutions ranging from $32^{3}$ to $256^{3}$. This method is often seen as the simplest way to deal with the (unknown) disk boundary conditions, but it has some limitations as discussed in past work (Pumir 1996; Umurhan \& Regev 2004; Fromang \& Papaloizou 2007). While Balbus et al. (1996); Hawley et al. (1999) did not use explicit viscosity, thereby making it difficult to directly define a Reynolds number, they estimated that their simulations achieved $R e \sim 10^{3}-10^{4}$. In their case, they did not find any evidence for a subcritical transition to turbulence for Keplerian flows, which they determined to be completely stabilized by the Coriolis force. The simulations of Lesur \& Longaretti (2005) at $N^{3}=128^{3}$ could achieve $R e \approx 4 \times 10^{4}$, according to their estimation. They concluded, on the other hand, that Keplerian like flows could exhibit turbulence, although the turbulence intensity may be too weak to account for the rate of energy dissipation observed. This has motivated us to experimentally explore Reynolds number up 
to $R e=4 \times 10^{6}$, a value that is beyond present computational efforts.

The overwhelming computational requirements needed to obtain larger Reynolds numbers motivate an alternate approach to explore the transport properties of Taylor-Couette flow through laboratory experiments, which can obtain much larger Reynolds numbers, as we present here. Depending upon the community, the strength of the turbulent transport is quantified in various ways. The most basic quantification (Wendt 1933) is the one through the torque $T$ which is necessary to keep the inner cylinder rotating at a given velocity. The dimensionless version thereof is $G=T /\left(L_{\text {mid }} \rho v^{2}\right)$, where $\rho$ is the fluid density, $v$ its kinematic viscosity and $L_{\text {mid }}$ the effective length of the cylinders (Paoletti \& Lathrop 2011). Eckhardt et al. (2007b) and van Gils et al. (2011b) used $N u_{\omega}=G / G_{\text {lam }}$, where $G_{\text {lam }}$ is the laminar torque, to quantify the angular velocity flux from the inner to the outer cylinder, in order to highlight the analogy between TaylorCouette and Rayleigh-Bénard flow (Dubrulle \& Hersant 2002; Eckhardt et al. 2007b,a). The engineering community prefers the drag-coefficient $c_{\mathrm{f}}=G / \operatorname{Re}^{2}$, where $R e=\frac{2}{1+\eta}\left|\eta R e_{2}-\operatorname{Re}_{1}\right|$ and $\eta=a / b$ is the radius ratio and $R e_{1 / 2}$ the Reynolds number of the inner/outer cylinder. In the astrophysics community, the same information is often expressed in terms of the $\beta$ coefficient, defined by $\beta=2 G \eta^{2} /\left(\pi(1-\eta)^{4} R e^{2}\right)=2 \eta^{2} c_{\mathrm{f}} /\left(\pi(1-\eta)^{4}\right)$, which can be interpreted as a dimensionless turbulent viscosity.

By reexamining previous data obtained by Wendt (1933) and Taylor (1936), Richard \& Zahn (1999) showed that the $\beta$-parameter is on the order of $\beta_{\mathrm{RZ}} \sim 10^{-5}$ for Keplerian disks. A more complete analysis, including results of new experiments on a classical Taylor-Couette flow from Richard (2001), led to the same conclusion (Dubrulle et al. 2005). In contrast, an original experiment, built in Princeton (Ji et al. 2006) so as to minimize end effects, measured a $\beta$ coefficient through radial Reynolds stresses and obtained a value smaller by more than an order of magnitude $\beta_{\mathrm{P}} \sim 7 \times 10^{-7}$. Furthermore, the Princeton experiments could not distinguish the angular momentum transport in Keplerian flows from their measurements in solid-body rotation, where the cylinders rotate together thereby resulting in zero angular momentum transport.

In the present contribution, we report precise global angular momentum transport (torque) measurements in two independent experiments performed at very large Reynolds number and compute the resulting $\beta$ coefficient for different rotation profiles. To further study the scaling of angular momentum transport in rotating shear flows, we compare our measurements to those of Wendt (1933), Taylor (1936) and Richard (2001), Dubrulle et al. (2005). We find a universal scaling of the torque for various rotation profiles that captures the effects of the various geometries used in the experiments summarized here.

\section{Apparatus and experimental details}

\subsection{Apparatus: generalities}

The experiments presented here all examine the transport of angular momentum (torque) in classical Taylor-Couette flow. Figure 1 shows a schematic representation of our experiments. The fluid is contained between concentric cylinders of radii $a<b$. The inner (outer) cylinder rotates at an angular velocity of $\Omega_{1}\left(\Omega_{2}\right)$. The overall height of the fluid contained between the axial boundaries is given by the length $L$. The geometry of a particular Taylor-Couette flow apparatus is often defined in dimensionless form by the radius ratio $\eta \equiv a / b$ and the aspect ratio $\Gamma \equiv L /(b-a)$.

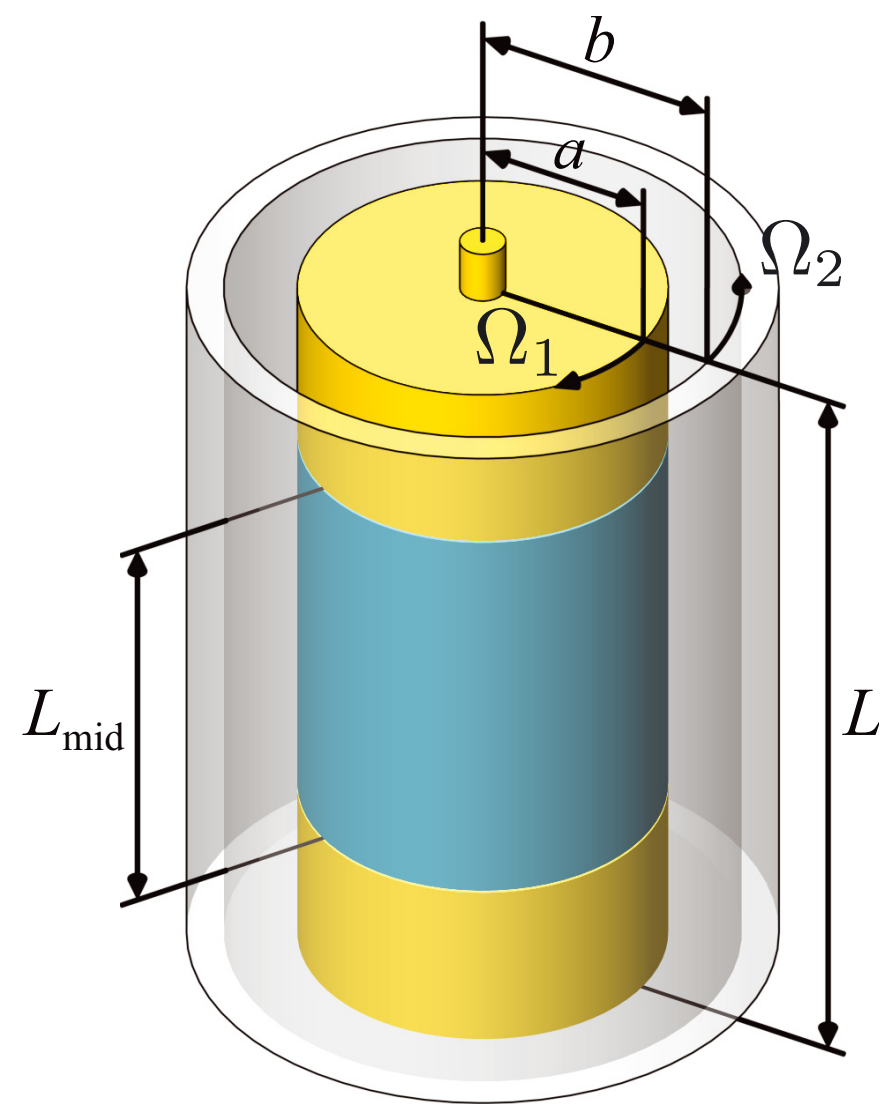

Fig. 1. Taylor-Couette flow may be used to study angular momentum transport in rotating shear flows. The fluid is sheared between two independently rotating cylinders of radii $a<b$ that rotate at angular velocities $\Omega_{1}$ and $\Omega_{2}$ for the inner and outer cylinders, respectively. Although the total fluid height is given by $L$, some experiments only measure the contribution to the torque over a central section of length $L_{\text {mid }}$ to minimize end effects, as in (Lathrop et al. 1992b,a; Lewis \& Swinney 1999; van den Berg et al. 2003, 2005, 2007; Paoletti \& Lathrop 2011; van Gils et al. 2011b).

The ideal Couette flow is infinite in axial extent. As such, several methods have been employed to handle the finite-size effects that are present in any laboratory experiment. The most common case has the axial boundaries rotate with one of the two cylinders. In other experiments, the axial boundaries are divided at the mid-gap and have the inner (outer) portion rotate with the inner (outer) cylinder. This split-ring approach has been further developed to allow for pairs of rings that can independently rotate with respect to the cylinders. Allowing the axial boundaries to independently rotate aids in the suppression of finite-size effects (Ji et al. 2006; Schartman et al. 2009; Burin et al. 2010).

The data that we analyze and present stems from several different experiments, which are summarized in Table 1 . The early experiments by Wendt (1933) measured the torque required to drive the inner cylinder for three values of $\eta$. The system had a free surface at the top, although we quote an effective aspect ratio defined using the axial length of the apparatus. The data from Taylor (1936) determined the scaling of the torque for several values of $\eta$ with the inner cylinder stationary $\left(\Omega_{1}=0\right)$. Richard's experiments (Richard 2001; Dubrulle et al. 2005) did not directly measure the torque. Instead, critical Reynolds numbers for various transitions were observed and the analysis presented by Dubrulle et al. (2005) was used to estimate the corresponding torque. We do not present data from the experiments of Ji et al. (2006), but rather use their reported measurements as a basis for 
Table 1. Parameters used in the experiments discussed here.

\begin{tabular}{lccccccc}
\hline \hline Experiment & $b(\mathrm{~cm})$ & $\eta$ & $\Gamma$ & $\max \left(R e_{1}\right)$ & $\max \left(\operatorname{Re}_{2}\right)$ & $\min \left(R_{\Omega}\right)$ & $\max \left(R_{\Omega}\right)$ \\
\hline Wendt (1933) & 14.7 & 0.68 & 10.64 & $8.47 \times 10^{4}$ & $1.24 \times 10^{5}$ & -0.71 & 0.47 \\
Wendt (1933) & 14.7 & 0.85 & 22.93 & $4.95 \times 10^{4}$ & $5.83 \times 10^{4}$ & -0.80 & 0.18 \\
Wendt (1933) & 14.7 & 0.935 & 52.63 & $2.35 \times 10^{4}$ & $2.52 \times 10^{4}$ & -0.42 & 0.11 \\
Taylor (1936) & 4.055 & $0.79-0.973$ & $100-768$ & 0 & $1.08 \times 10^{5}$ & 0.03 & 0.27 \\
Richard (2001) & 5.00 & 0.72 & 27.18 & $9.50 \times 10^{4}$ & $1.30 \times 10^{5}$ & -1.43 & -0.94 \\
Richard (2001) & 5.00 & 0.72 & 27.18 & $9.50 \times 10^{4}$ & $1.30 \times 10^{5}$ & 0.38 & 0.58 \\
Ji et al. (2006) & 20.3 & 0.35 & 2.1 & $1.20 \times 10^{6}$ & $4.50 \times 10^{5}$ & -1.67 & -1.08 \\
Maryland & 22.09 & 0.725 & 11.47 & $3.70 \times 10^{6}$ & $1.50 \times 10^{6}$ & -2.04 & 1.46 \\
Twente & 27.9 & 0.716 & 11.68 & $2.00 \times 10^{6}$ & $1.40 \times 10^{6}$ & -0.74 & 0.17 \\
\hline
\end{tabular}

Notes. The geometry of each apparatus is given by the outer cylinder radius $b$, the radius ratio $\eta=a / b$ and the aspect ratio $\Gamma=L /(b-a)$. The range of turbulence intensities are governed by the inner and outer cylinder Reynolds numbers $R e_{1}$ and $R e_{2}$. The effects of rotation are parameterized by the rotation number $R_{\Omega}$ introduced by Dubrulle et al. (2005).

comparison, as detailed in Sect. 4. The two most recent experiments by Paoletti \& Lathrop (2011) and van Gils et al. (2011b) are further described in the forthcoming sections.

\subsection{Apparatus: the Maryland experiment}

The experiments presented by Paoletti \& Lathrop (2011), henceforth referred to as the Maryland experiment, are conducted in the apparatus constructed by Lathrop et al. (1992b,a), which was modified to allow the outer cylinder to rotate independently. The outer cylinder is an anodized aluminum cylinder with the same physical dimensions as in Lathrop et al. (1992b,a); Lewis \& Swinney (1999), specifically $b=22.085 \mathrm{~cm}$ and a working fluid height $L=69.50 \mathrm{~cm}$. The inner cylinder is stainless steel with a radius $a=16.000 \mathrm{~cm}$ yielding a radius ratio $\eta=0.7245$ and an aspect ratio $\Gamma=11.47$. The inner cylinder is rotated up to $\Omega_{1} / 2 \pi=20 \mathrm{~s}^{-1}$ while the outer cylinder may be rotated in either direction up to $\left|\Omega_{2} / 2 \pi\right|=10 \mathrm{~s}^{-1}$. Both angular velocities are measured by shaft encoders and controlled to within $0.2 \%$ of the set value.

The axial boundaries in the Maryland experiment rotate with the outer cylinder. To reduce end effects in the torque measurements the inner cylinder is divided axially into three sections of length $15.69,40.64$ and $15.69 \mathrm{~cm}$ (see schematics in Lathrop et al. 1992b,a). Only the central section of length $L_{\text {mid }}=40.64 \mathrm{~cm}$ (see Fig. 1) senses the torque of the fluid as described in Lathrop et al. (1992b,a). The regions of the flow within 2.58 gap widths from each of the axial boundaries, where secondary circulation setup by finite boundaries is strongest, are avoided in the torque measurements.

The desired accuracy of our measurements requires that the temperature of the water be precisely controlled. In contrast to prior experiments (Lathrop et al. 1992b,a; Lewis \& Swinney 1999; van den Berg et al. 2003, 2005, 2007; van Gils et al. $2011 \mathrm{~b}$ ) where the system was cooled at the axial boundaries, we control the temperature of the water by heating and cooling the outer cylinder. This allows the working fluid to be temperaturecontrolled along the entire axial length of the experiment, yielding a 6.5 fold increase in the temperature-controlled surface area. This is particularly important for Rayleigh-stable flows, where mixing is greatly reduced. In our experiments the temperature is $50 \pm 0.02{ }^{\circ} \mathrm{C}$ yielding a kinematic fluid viscosity of $v=5.5 \times 10^{-3} \mathrm{~cm}^{2} / \mathrm{s}$, except for $\operatorname{Re}>2 \times 10^{6}$ where $T=90^{\circ} \mathrm{C}$ and $v=3.2 \times 10^{-3} \mathrm{~cm}^{2} / \mathrm{s}$.

\subsection{Apparatus: the Twente experiment}

The Twente turbulent Taylor-Couette (called $\mathrm{T}^{3} \mathrm{C}$ ) facility, here referred to as the Twente experiment, is described in great detail in van Gils et al. (2011a). In short, the working fluid height is $L=$ $92.7 \mathrm{~cm}$, has an inner radius of $a=20.0 \mathrm{~cm}$ and an outer radius of $b=27.94 \mathrm{~cm}$. The maximum inner and outer angular velocities are $\Omega_{1} / 2 \pi=20 \mathrm{~Hz}$ and $\left|\Omega_{2} / 2 \pi\right|=10 \mathrm{~Hz}$, respectively. The system is fully temperature controlled through cooling of the upper and lower end plates, which co-rotate with the outer cylinder. The system is operated with water at a temperature of $20^{\circ} \mathrm{C}$, resulting in a kinematic viscosity of $v=1.04 \times 10^{-2} \mathrm{~cm}^{2} / \mathrm{s}$. The torque is measured over the middle part of the inner cylinder of height $L_{\text {mid }}=53.6 \mathrm{~cm}$ to minimize the influence of the endplates (similar to Lathrop et al. 1992b,a). The torque is measured by a load cell imbedded inside the middle section of the inner cylinder in both the Twente and Maryland experiments, in contrast to measuring the torque on the drive shaft that would be affected by mechanical seals in the end plates or by velocimetry measurements, as in the Princeton experiments ( $\mathrm{Ji}$ et al. 2006; Schartman et al. 2009; Burin et al. 2010; Schartman et al. 2012).

\subsection{Control parameters}

The analysis of our results is simplified by a proper choice of the control parameters. As in Dubrulle et al. (2005), we use the following three dimensionless control parameters, which fully describe the geometry and specify the state of the dynamical Taylor-Couette system:

$$
\begin{aligned}
R e & =\frac{2}{1+\eta}\left|\eta R e_{2}-R e_{1}\right| \\
R_{\Omega} & =(1-\eta) \frac{R e_{1}+R e_{2}}{\eta R e_{2}-R e_{1}}, \\
R_{C} & =\frac{1-\eta}{\eta^{1 / 2}},
\end{aligned}
$$

where $R e_{1}=a \Omega_{1} d / v$ and $R e_{2}=b \Omega_{2} d / v$ are the Reynolds number of the inner and outer cylinder and $d=(b-a)$ is the gap width.

The above control parameters have been introduced so that their definitions apply to rotating shear flows in general and not strictly only to the Taylor-Couette geometry. It is very easy in this formulation to relate the Taylor-Couette flow to the shearing sheet (plane Couette flow with rotation), by considering the limit $R_{\mathrm{C}} \rightarrow 0$. The linear stability properties of the fluid can also 
A\&A 547, A64 (2012)

Table 2. Taylor-Couette control parameters related to some astrophysical disks, as computed by Hersant et al. (2005).

\begin{tabular}{lccccccc}
\hline \hline Central object & $b$ (a.u.) & $\eta$ & $\Gamma$ & $\max \left(R e_{1}\right)$ & $\max \left(R e_{2}\right)$ & $R_{\Omega}$ & $\dot{M} / \dot{M}_{0}$ \\
\hline T Tauri & 1000 & 0 & 0.94 & $10^{12}$ & $10^{14}$ & -1.33 & $10^{-5}-10^{-2}$ \\
Fu Ori & 5 & 0 & 0.94 & $10^{13}$ & $10^{15}$ & -1.33 & $0.1-1$ \\
\hline
\end{tabular}

Notes. Here, $b$ is the disk's outer radius, and $\dot{M}$ the accretion rate.

be neatly recast using these control parameters. In the inviscid limit $(R e \rightarrow \infty)$, and for axisymmetric disturbances, the linear stability properties of the flow are governed by the Rayleigh criterion, namely that the fluid is stable if the Rayleigh discriminant is everywhere positive:

$\frac{\Omega}{r} \partial_{r} L(r)>0$,

where $L(r)=r^{2} \Omega(r)$ is the specific angular momentum. Applying this criterion to the laminar profile leads to

$\left(R_{\Omega}+1\right)\left(R_{\Omega}+1-a b / r^{2}\right)>0$.

Since $a b / r^{2}$ varies between $1 / \eta$ and $\eta$, one obtains that in the inviscid limit, the flow is unstable against infinitesimal axisymmetric disturbances when $R_{\Omega}^{\infty-}<R_{\Omega}<R_{\Omega}^{\infty+}$, where $R_{\Omega}^{\infty-}=-1$, respectively $R_{\Omega}^{\infty+}=1 / \eta-1$, are the marginal stability thresholds in the inviscid limit (superscript $\infty$ ) in the cyclonic case $\left(R_{\Omega}>0\right.$, subscript + ), and anticyclonic case $\left(R_{\Omega}<0\right.$, subscript -$)$. This means that, in the anticyclonic case, any flow such that $R_{\Omega}<-1$ is linearly stable against axisymmetric disturbances. However, it may be non-linearly stable or unstable against non-axisymmetric disturbances, as found in the experiments described here.

In the present paper, we determine the intensity of the momentum transport by measuring the torque required to drive the inner cylinder for a given set of parameters $\left(\operatorname{Re}, R_{\Omega}\right)$. Measurements described here suggest that at large enough Reynolds number, the nondimensional torque $G=T /\left(\rho v^{2} L_{\text {mid }}\right)$, where $T$ is the torque, $\rho$ the fluid density, $v$ the kinematic viscosity and $L_{\text {mid }}$ is defined in Fig. 1, varies approximately as $G \sim R e^{\alpha}$ with $1.75<\alpha(R e)<1.85$, even for Rayleigh-stable flows. While this scaling exceeds the laminar case $(G \sim R e)$, we note that these exponents are less than a simple dimensional argument would give: Such an argument would say that in a fully turbulent regime the transport properties could not depend on the molecular viscosity, which would imply that the quantity $c_{\mathrm{f}} \sim G / R e^{2}$ is Reynolds number independent. The deviations from $G \sim R e^{2}$ mean that the viscosity is always relevant for the transport, due to its dominance in the boundary layers. Only when the boundary layers are sufficiently disturbed by introducing wall roughness does one obtain $G \sim R e^{2}$, as we have shown in our previous work (van den Berg et al. 2003).

\subsection{Astrophysically relevant quantity}

In the astrophysical context, one often considers asymptotic angular velocity profiles of the form $\Omega(r) \sim r^{-q}$ where $q$ characterizes the flow. In that case $q=-\partial \ln \Omega / \partial \ln r=-2 / R_{\Omega}$, which relates astrophysical profiles in the control parameters space of Taylor-Couette flows. For Keplerian flow, $q=3 / 2$ resulting in $R_{\Omega}=-4 / 3$, which is an example of a linearly stable, anticyclonic flow. While Keplerian profiles cannot be precisely achieved by Taylor-Couette flows, states with $R_{\Omega}<-1$ are all linearly stable, anticyclonic flows that may be used to approximate astrophysical settings. We will refer to cases with $R_{\Omega}<-1$ as quasi-Keplerian, as in Ji et al. (2006).
Astrophysical disks are very wide with $a \ll b$. Therefore, their geometrical global value of $R_{\mathrm{C}} \rightarrow \infty$. In a local description of disks, one often considers the shearing sheet approximation, in which $\eta \rightarrow 1$, so that $R_{\mathrm{C}} \rightarrow 0$, as in plane-Couette flow. Here, we focus on global properties and consider only the limit $\eta \rightarrow 0$ applicable to disks in the discussion. As a result of their astronomical size, the Reynolds numbers associated with accretion disks are very large. To give a few specific examples, parameters for typical T Tauri and Fu Ori stars have been computed by Hersant et al. (2005) and are given in Table 2.

In disks, angular momentum transport intensity is quantified through the accretion rate $\dot{M}$. In fact, as was shown in (Dubrulle et al. 2005; Hersant et al. 2005), this rate is directly related to the turbulent viscosity and the dimensionless torque. This concept may be understood in the following way: pressure gradients substantially balance centrifugal forces that develop in (incompressible) laboratory flows. Keplerian disks, however, balance the centrifugal force with gravitational attraction. Thus when angular momentum is transported radially outward, accretion disk matter responds by falling radially inward at the rate $\dot{M}$. The flux of angular momentum, which is proportional to $G$, is thereby related to the accretion rate $\dot{M}$ by (Dubrulle et al. 2005; Hersant et al. 2005),

$$
\frac{G}{R e^{2}}=\frac{\pi}{2 R_{C}^{4}} \beta=\frac{\dot{M}}{\dot{M}_{0}}
$$

where $\beta$ is a turbulent viscosity parameter such that $v_{\mathrm{t}}=\beta S r^{2}$, with $S$ a velocity shear, and $\dot{M}_{0}$ is an effective accretion rate given by the mean surface density $\bar{\Sigma}$, the disk inner radius $a$, mean radius $\bar{r}=(a+b) / 2$, the mean angular velocity $\bar{\Omega}$ and the mean disk height $\bar{H}$ through

$\dot{M}_{0}=\bar{\Sigma} a \bar{r} \bar{\Omega}\left(\frac{\bar{H}}{\bar{r}}\right)^{4}$.

As such, measurements of $G / R e^{2}$ in laboratory experiments may be used to estimate the hydrodynamical contribution to angular momentum transport in accretion disks.

\section{Results}

We study the scaling of the dimensionless torque $G$ over a range of Reynolds numbers for various constant rotation numbers (see Fig. 2), spanning a wide variety of flow states, including linearly stable cyclonic and anticyclonic flows as well as linearly unstable states. Esser \& Grossmann (1996) derived an analytical expression that approximates the boundary for linear stability for all radius ratios and cylinder rotation rate ratios. Their result for the critical Reynolds number $R e_{\mathrm{c}}$ may be expressed using the above control parameters, as in Eqs. (16) and (17) in Dubrulle et al. (2005):

$\operatorname{Re}_{\mathrm{c}}^{2}\left(R_{\Omega}+1\right)\left(R_{\Omega}+1-\frac{1}{\eta x(\eta)^{2}}\right)=-1708\left(\frac{1-\eta}{2 \eta[x(\eta)-1]}\right)^{4}$, 
M. S. Paoletti et al.: Angular momentum transport and turbulence in Keplerian flows

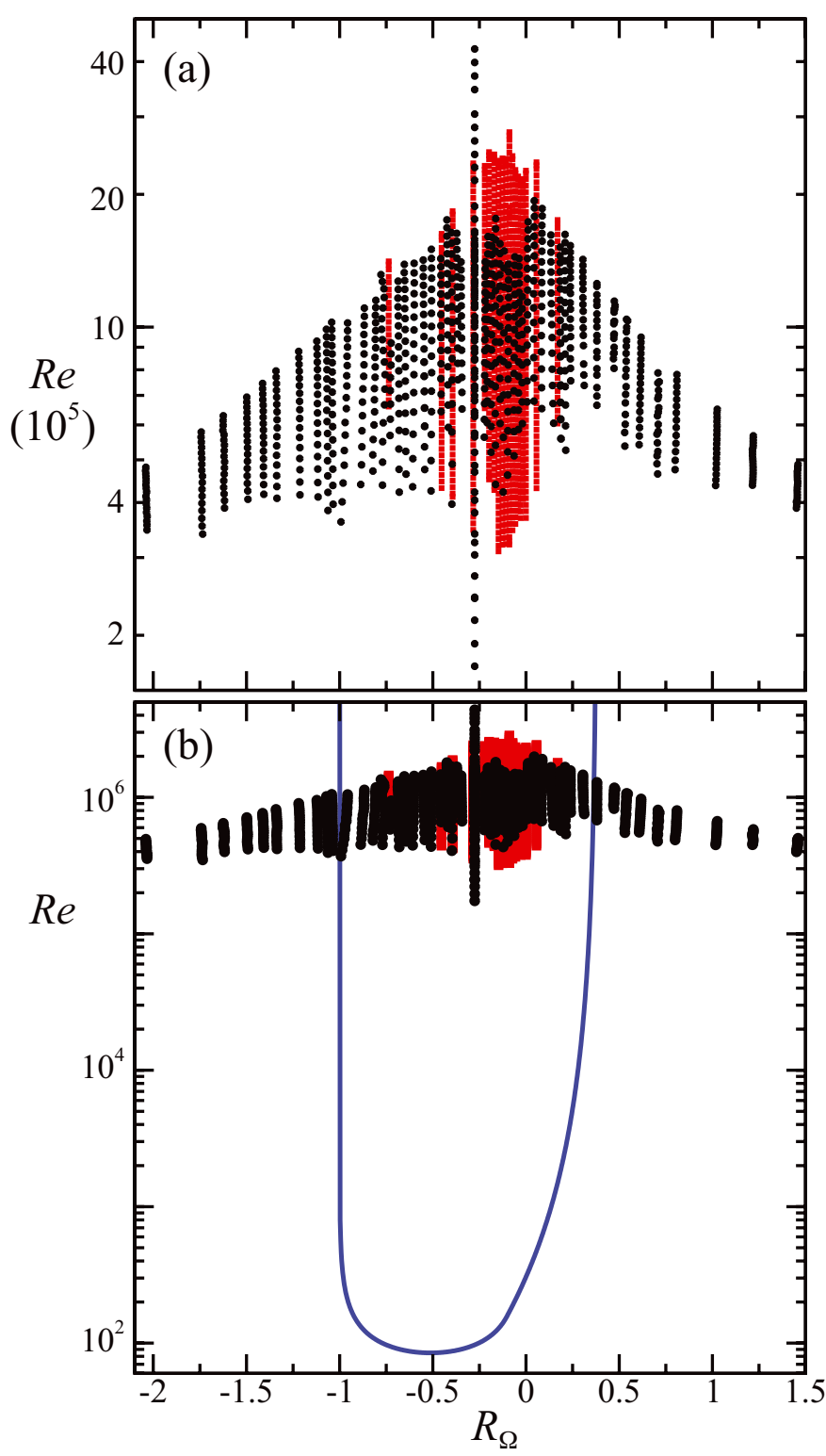

Fig. 2. a) Our experiments span a large range of the Reynolds number $R e$, which controls the turbulence intensity, and the rotation number $R_{\Omega}$, introduced by Dubrulle et al. (2005), which compares shear to overall rotation. The rotation number can be used to distinguish between Rayleigh-stable flows $\left(R_{\Omega} \leq-1\right.$ or $\left.R_{\Omega} \geq(1-\eta) / \eta\right)$ and those that are linearly unstable $\left(-1<R_{\Omega}<(1-\eta) / \eta\right)$. b) All of our measurements for $-1<R_{\Omega}<0.38$ are well above the linear stability curve (blue line) described by Esser \& Grossmann (1996) and given by Eq. (8). The data are taken from separate experiments by van Gils et al. (2011b) (red squares) and (Paoletti \& Lathrop 2011) (black circles).

with

$x(\eta)=1+\frac{1-\eta}{2 \eta} \Delta\left(\epsilon(\eta) \frac{d_{n}}{d}\right)$,

$\frac{d_{n}}{d}=\frac{\eta}{1-\eta}\left(\frac{1}{\sqrt{\eta\left(R_{\Omega}+1\right)}}-1\right)$,

$\epsilon(\eta)=(1-\eta)\left(\sqrt{\frac{(1+\eta)^{3}}{2(1+3 \eta)}}-\eta\right)^{-1}$,

where $\Delta(x)$ is a function equal to $x$ if $x<1$ and equal to 1 if $x>1$. Equation (8) may be used to determine the stability

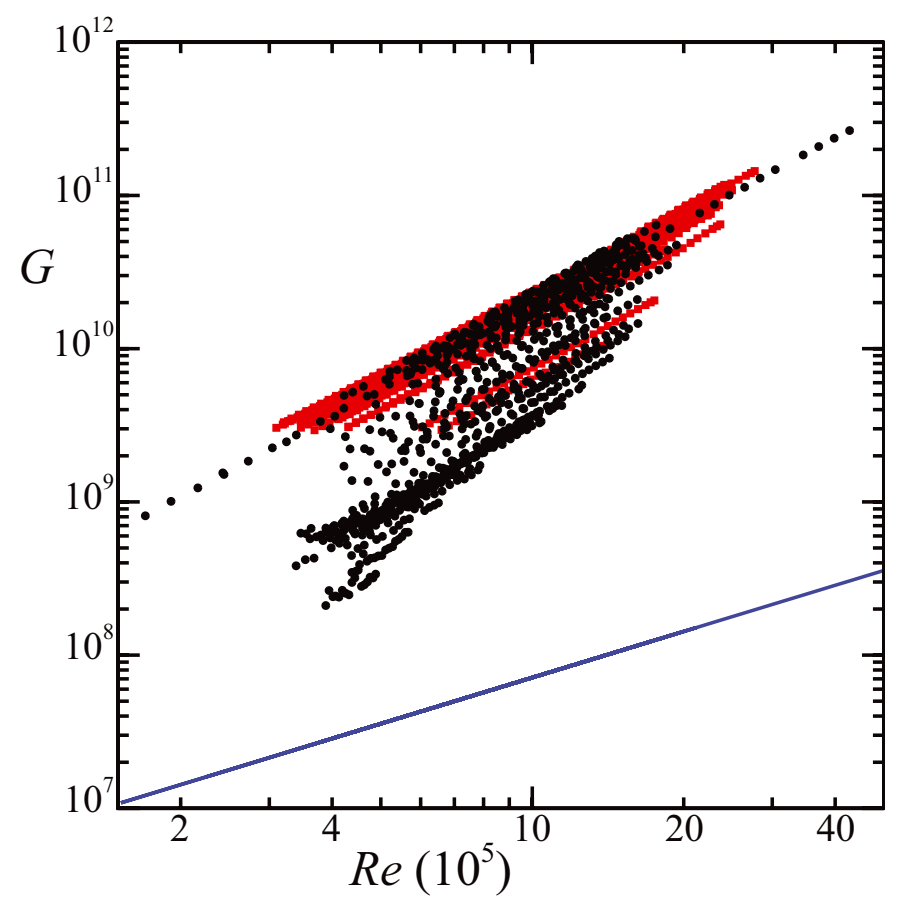

Fig. 3. The scaling of the dimensionless torque $G$ with the Reynolds number $R e$ is independent of the rotation number $R_{\Omega}$, however the amplitude varies as in Paoletti \& Lathrop (2011); van Gils et al. (2011b). The measurements by Paoletti \& Lathrop (2011) are in black circles while the data from van Gils et al. (2011b) are shown by red squares. The thick blue line indicates the theoretical value for purely laminar flow, where angular momentum is transported only by molecular viscosity.

boundary for the experiments described in Table 1. As shown in Fig. 2b, all of our measurements for $-1<R_{\Omega}<0.38$ are orders of magnitude above the laminar stability boundary. For $R_{\Omega}<-1$ or $R_{\Omega}>0.38$ the system is linearly stable for our radius ratios, although it may be possible for the system to undergo a subcritical transition to turbulence. Corresponding torque measurements from the Twente experiment are shown as red squares in Fig. 3 while the data from Maryland are represented by black circles.

\subsection{Existence of turbulence}

In laminar flow, angular momentum is transported only by molecular viscosity, so that the dimensionless torque $G$ is proportional to $R e$, with the prefactor depending upon $R_{\Omega}$. In Fig. 3, we show the expected laminar angular momentum transport (blue line) for the range of $R e$ explored in this study for the case $\Omega_{2}=0$. As can be seen all the torque measurements considered differ from the theoretical expectation for laminar flow in two ways: ( $i$ ) they increase faster than $R e$ and (ii) they are much higher than the theoretical laminar value. This is an indication that the fluid is turbulent, with enhanced angular momentum transport in all the cases reported here. Note that these cases encompass several situations with $R_{\Omega}<-1$ (linearly stable anticyclonic flow) and $R_{\Omega}>0.4$ (linearly stable cyclonic flow) in addition to many unstable states.

In particular, (approximate) Keplerian flows at $R_{\Omega}=-4 / 3$ are turbulent at $\operatorname{Re} \sim 10^{5}$ in our experiments. This is in agreement with direct, visual observations of Richard (2001), performed at somewhat lower Reynolds number. We argue that this indicates that quasi-Keplerian flows can efficiently transport angular momentum in spite of their linear stability. However, 
the nature of this nonlinear instability remains unclear. We suggest that systematically perturbing quasi-Keplerian flow states at lower values of $R e$ while measuring the torque, as in the experiments by Taylor (1936), could aid in the description of this likely nonlinear instability.

\subsection{Enhancement of momentum transport}

We now try to quantify the enhancement of angular momentum transport with respect to its laminar value, as a function of both $R e$ and $R_{\Omega}$. As in Paoletti \& Lathrop (2011); van Gils et al. (2011b), the torque at a given $R e$ may be either increased or reduced depending upon $R_{\Omega}$; the Reynolds number alone is insufficient to describe the transport.

\subsubsection{Variation with $R_{\Omega}$}

Paoletti \& Lathrop (2011) observed that variation of the torque at a given $R e$ shows a pronounced maximum as a function of $R_{\Omega}$. An analogous dependence of the amplitude of an effective power-law scaling was also observed by van Gils et al. (2011b). To determine the effects of global rotation, we normalize $G$ for each $R e$ by $G\left(R e, R_{\Omega}=0\right)$ (Dubrulle et al. 2005), which we denote as $G_{0}$. This normalization is different from Fig. 16 in Dubrulle et al. (2005) where $G$ is normalized by $G_{\infty} \equiv G\left(\operatorname{Re}, \Omega_{2}=0\right)$. The dependence of $G / G_{0}$ on $R_{\Omega}$ is shown in Fig. 5 with the data from Maryland shown as black circles and Twente in red squares.

The agreement between the Twente and Maryland measurements is an important check, but not surprising given that the experiments have very similar geometries, measurement techniques and control parameters (see Table 1). Therefore, it remains unclear if the scaling of the normalized torque with $R_{\Omega}$ is universal or if it depends upon other parameters, such as the radius ratio $\eta$. While the Twente experiment is capable of examining other values of $\eta$ in future measurements, we may compare our results to previous experiments by Wendt (1933), Taylor (1936) and Richard (2001), which were analyzed by Dubrulle et al. (2005). The parameters of these past experiments are also summarized in Table 1. The measurements of Wendt (triangles), Taylor (orange diamonds) and Richard (magenta squares) are also shown in Fig. 4a.

As can be seen in Fig. 4, the dependence of the normalized torque $G / G_{0}$ on $R_{\Omega}$ for various radius ratios in the range $0.68 \leq \eta \leq 0.973$ collapse well. The data appear to follow distinct approximate scalings given by

$f\left(R_{\Omega}\right) \approx \begin{cases}0.18 \pm 0.06 & : R_{\Omega} \leq-1 \\ 1.79 R_{\Omega}+0.005 \mathrm{e}^{-4.35 R_{\Omega}}+1.59 & :-1<R_{\Omega} \leq-0.10 \\ -7.35 R_{\Omega}+1.49 \mathrm{e}^{2.28 R_{\Omega}}-0.51 & :-0.10<R_{\Omega}<0.32 \\ -0.13 R_{\Omega}+0.26 & : R_{\Omega} \geq 0.32\end{cases}$

Given the rather different span of $R e$ in each experiment and the varying measurement techniques, it is not surprising that a few outliers remain. For example, the values of $G / G_{0}$ from Richard's experiments (Richard 2001) (magenta squares) were computed using theoretical predictions regarding the correlation between the torque and critical numbers for stability, as described in Dubrulle et al. (2005). The data from Wendt (1933), Taylor (1936), Paoletti \& Lathrop (2011) and van Gils et al. (2011b) are direct measurements of the torque, albeit with different apparati and measurement techniques.
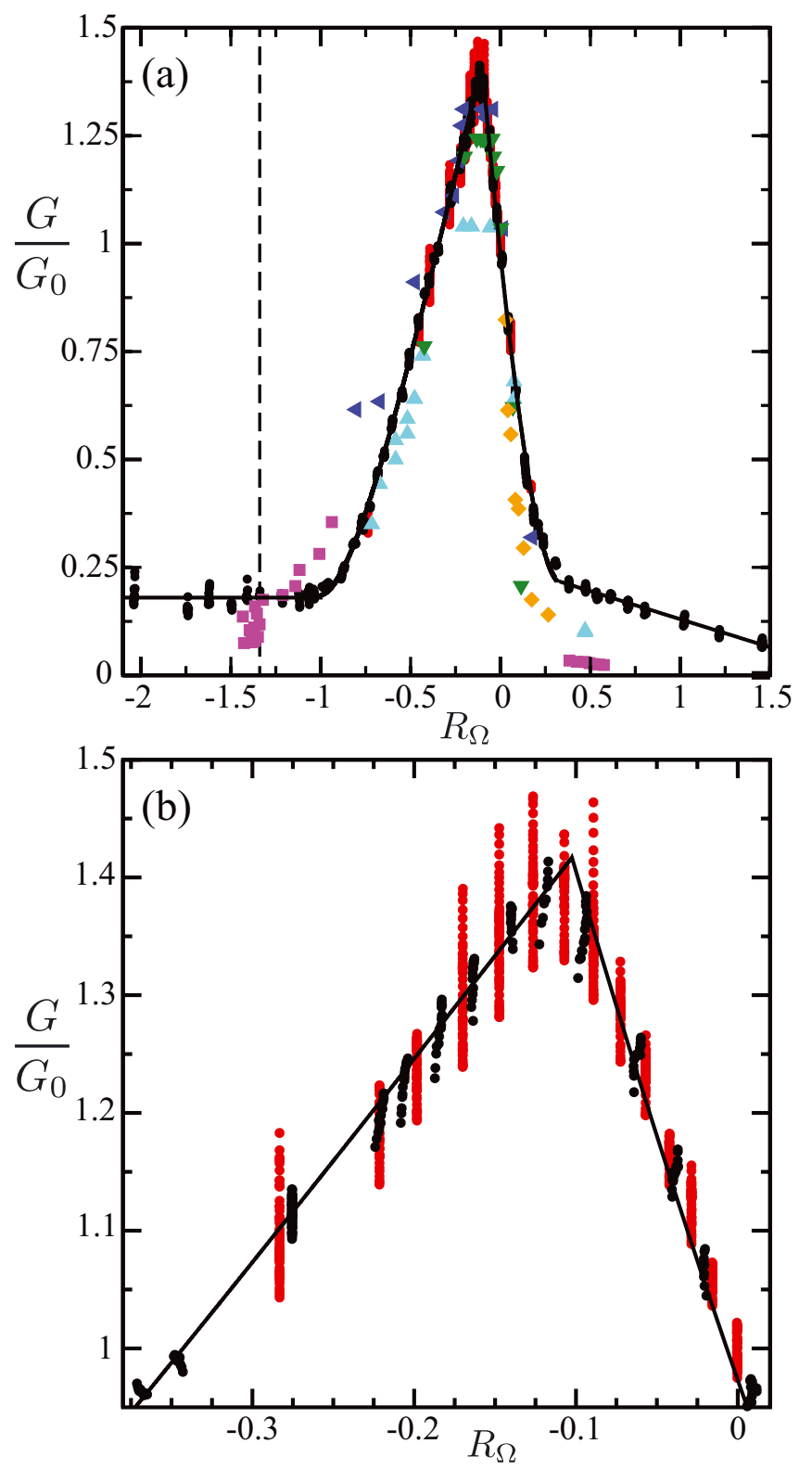

Fig. 4. a) The dimensionless torque $G$ normalized by $G_{0} \equiv G\left(R e, R_{\Omega}=\right.$ 0 ) for different values of the radius ratio $\eta$ nicely collapses when plotted as function of $R_{\Omega}$. The data from Wendt (1933) are shown as cyan $(\eta=0.68)$, blue $(\eta=0.85)$ and green $(\eta=0.935)$ triangles. Additional radius ratios are examined using the data from Taylor (1936), shown as orange diamonds, Richard (Richard 2001; Dubrulle et al. 2005), given by magenta squares, Paoletti \& Lathrop (2011) indicated by black circles and van Gils et al. (2011b) by red circles. The solid lines are fits given by Eq. (12). Keplerian flow states are indicated by the dashed line at $R_{\Omega}=-4 / 3$. In b) we focus on the peak of the curve shown in a), but only present the data from Twente (van Gils et al. 2011b) (red) and Maryland (Paoletti \& Lathrop 2011) (black). The solid line is the fit (12).

\subsubsection{Variation with Reynolds number}

The fits given in Eq. (12) may be used to compensate the torque to account for the effects of global rotation. Figure 5 shows the dimensionless torque $G$ compensated by the fit $f\left(R_{\Omega}\right)$ given in Eq. (12) as a function of $R e$. The data from Twente are shown as red squares while those of Maryland are given by black circles. In both cases, the data agree well with the best fit given by Lewis $\&$ Swinney (1999) for the case $\Omega_{2}=0$ (blue line). This indicates 


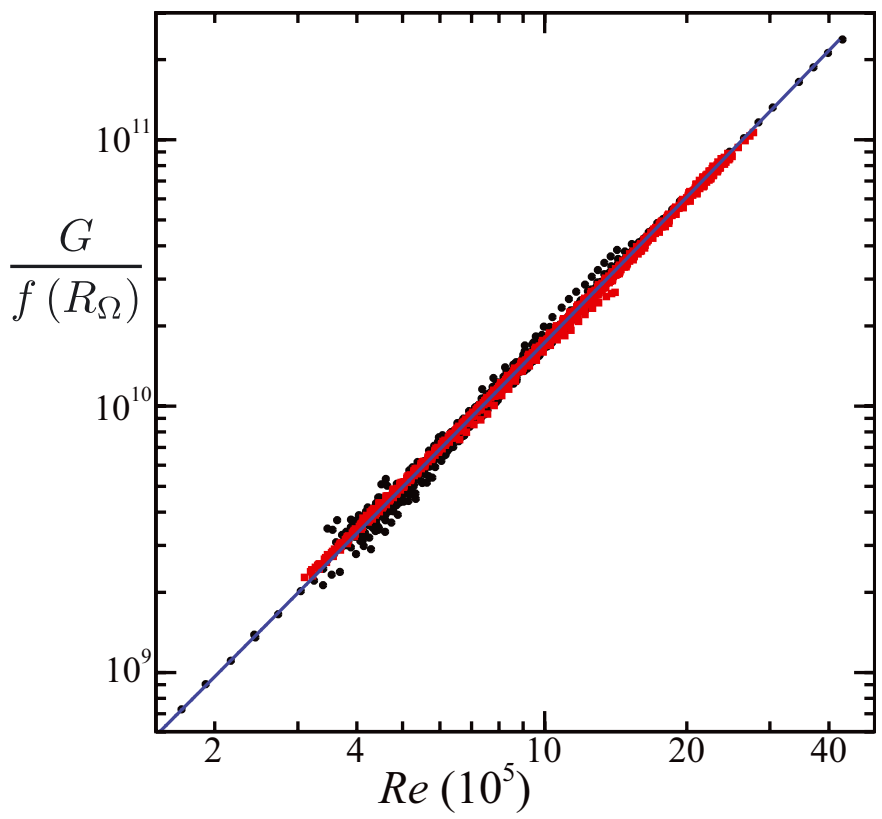

Fig. 5. The dimensionless torque $G$ may be compensated by the fit $f\left(R_{\Omega}\right)$ given by Eq. (12). The data from both Paoletti \& Lathrop (2011) (black circles) and van Gils et al. (2011b) (red squares) agree with the best fit from Lewis \& Swinney (1999) for the case where the outer cylinder was stationary (blue line).

that the amplitude of the torque is affected by global rotation, as measured by $G / G_{0}$, while the scaling with $R e$ seems to be independent of $R_{\Omega}$ in the regime of our investigation. Therefore the torque may be approximately factorized and written as

$G=f\left(R_{\Omega}\right) g(R e)$

where $f\left(R_{\Omega}\right)$ is measured in Fig. 4 and fit by Eq. (12) and our measurements of $g(R e)$, shown in Fig. 5, agree with previous measurements, and may be fit by effective power-laws or logarithmically varying functions such as the Prandtl-von Kármán form (Lathrop et al. 1992b,a; Lewis \& Swinney 1999).

\subsubsection{Comments}

In addition to the observed data collapse, other interesting features arise in the normalized torque scaling with $R_{\Omega}$. The data from Taylor's experiments (Taylor 1936) were obtained with a stationary inner cylinder $\left(\Omega_{1}=0\right)$, and the flow is therefore Rayleigh-stable. However, the data from Wendt (1933), Maryland (Paoletti \& Lathrop 2011) and Twente (van Gils et al. $2011 \mathrm{~b}$ ) over the same range of the rotation number, namely $0<R_{\Omega}<0.27$, are obtained with counter-rotating cylinders. This configuration is linearly unstable, yet the measured values of $G / G_{0}$ agree between all four experiments. We argue, as done by Taylor (1936), that the flow states in Taylor's experiments could not have been laminar and must have undergone a subcritical transition to turbulence.

Paoletti \& Lathrop (2011) observed that the torque scaling varied linearly with $R_{\Omega}$ in four regions of the parameter space corresponding to: region I: $R_{\Omega} \leq-1$, region II: $-1<R_{\Omega} \leq$ -0.10 , region III: $-0.10<R_{\Omega}<0.38$ and region IV: $0.38<R_{\Omega}$, where we have reordered the region numbers to correspond to increasing $R_{\Omega}$. Figure 4 shows that all of the data described here follow the region I-region II and region II-region III crossovers. The crossover between regions I and II corresponds to the
Rayleigh stability criterion for all values of $\eta$. The crossover between regions II and III occurs at the observed maximum in the normalized torque $G / G_{0}$ as a function of $R_{\Omega}$. While this maximum occurs for all of the Wendt (1933), Maryland (Paoletti \& Lathrop 2011) and Twente (van Gils et al. 2011b) datasets, there is no general theoretical prediction for the exact location of this maximum yet.

The last crossover observed by Paoletti and Lathrop, between regions III and IV, corresponded to $\Omega_{1}=0$ with $\Omega_{1}>0$ and $\Omega_{2}<0$ in region III (counter-rotation) to $\Omega_{1}>0$ and $\Omega_{2}>0$ in region IV (co-rotation). It is unclear whether this crossover is set by $R_{\Omega}=0.38$ or whether it always occurs at $R_{\Omega}\left(\Omega_{1}=0\right)$. The data from Taylor (1936) has $\Omega_{1}=0$ but given the values of $\eta$ used in those experiments $R_{\Omega}<0.38$. The Richard data for $R_{\Omega}>0.38$ fall below the observations by Maryland, although the scaling with $R_{\Omega}$ is similar. It is unclear if this disparity is a result of the analysis used to deduce the torques from Richard's measurements or the difference in the control parameters from the Maryland experiment (see Table 1). It would be fruitful to have independent, direct measurements of the torque in region IV to compare against those of Maryland at different radius ratios to better understand this final crossover.

The Richard and Maryland data both indicate that the observed torque for quasi-Keplerian flows states $\left(R_{\Omega} \leq-1\right)$ is approximately $14 \%$ of the maximum observed torque for a given Reynolds number. This is in contrast to the results of $\mathrm{Ji}$ et al. (2006), who deduced that such flow states are "essentially steady" and unable to transport angular momentum hydrodynamically. In addition to the different geometries between the experiments in Ji et al. (2006) and those of Richard and Maryland (see Table 1), the measurement techniques and finite-size effects were different between the experiments. This contrast is further discussed in Sect. 4. Clearly, additional independent measurements of the angular momentum flux for quasi-Keplerian flows could aid this debate, as recently discussed by Balbus (2011).

\subsubsection{Astrophysical implications}

As described in Sect. 2.5, the scaling of the torque with Reynolds number may be used to determine either the turbulent viscosity parameter $\beta$ or the accretion rate $\dot{M}$ through Eq. (6). Our measurements of $G / R e^{2}$, which is proportional to both $\beta$ and $\dot{M}$, are shown in Fig. 6a. The measurements from Twente are shown as red squares while those of Maryland are given by black circles. The data indicated by blue diamonds are quasi-Keplerian flow states $\left(R_{\Omega} \leq-1\right)$ from the Maryland experiment. The quantity $G / R e^{2}$ decreases slowly with $R e$ (only logarithmically, see Dubrulle et al. 2005; Hersant et al. 2005; Grossmann \& Lohse 2011) as expected since $G \propto R e^{\alpha}$ with $1.8<\alpha(R e) \leq 2.0$ for $10^{5}<R e<\infty$ (Lathrop et al. 1992b,a; Lewis \& Swinney 1999). The amplitude of $G / R e^{2}$ for a given $R e$ is determined by $R_{\Omega}$. The effects of global rotation may be accounted for by compensating the measurements of $G / R e^{2}$ by $f\left(R_{\Omega}\right)$ (see. Eq. (12)), as shown in Fig. 6b. As discussed in Dubrulle \& Hersant (2002), the scaling may be fit by a logarithmically decaying function, such as:

$\frac{G}{R e^{2}}=K_{7} \frac{\eta^{2}}{(1-\eta)^{3 / 2}} \frac{1}{\ln \left[\eta^{2}(1-\eta) R e^{2} / K_{8}\right]^{3 / 2}}$,

with the values chosen to be $K_{7}=0.4664$ and $K_{8}=10^{4}$ to describe the best fit for the torque scaling provided by Lewis \& Swinney (1999) for the case $\Omega_{2}=0$. An alternative logarithmic dependence between $G / R e^{2}$ and $R e$ has been suggested by 


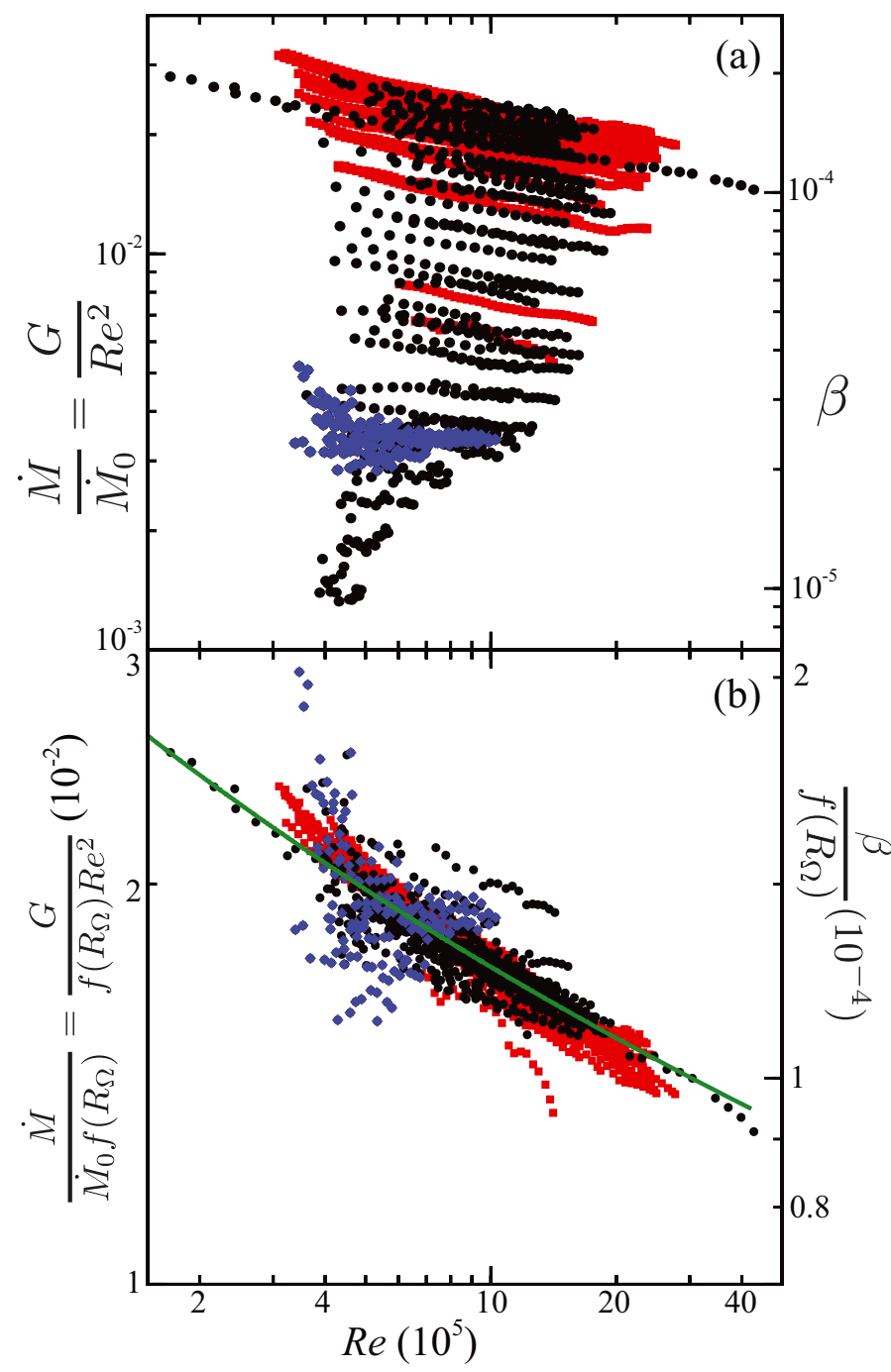

Fig. 6. The scaling of a) $G / R e^{2}$, which is proportional to the accretion rate $\dot{M}$ and viscosity parameter $\beta$, decays slowly with $R e$ while having an amplitude that varies with the rotation number $R_{\Omega}$ (see Fig. 4). b) The effects of global rotation can be accounted for by compensating $G / R e^{2}$ by the fits $f\left(R_{\Omega}\right)$ given in Eq. (12). The measurements from Twente are shown as red squares. The Maryland data are given by black circles, except for quasi-Keplerian flows $\left(R_{\Omega} \leq-1\right)$, which are indicated by blue diamonds. The green continuous line is the theoretical fit given in Eq. (14) normalized by $f\left(R_{\Omega}=-0.2755\right)$, which corresponds to $\Omega_{2}=0$.

Grossmann \& Lohse (2011) (for the analogous case of ultimate Rayleigh-Bénard flow), and it equally well fits the data.

The expected accretion rate, measured by $G / R e^{2}=3.3 \times$ $10^{-3}$, for quasi-Keplerian flows (blue diamonds in Fig. 6) is approximately $14 \%$ of the maximum accretion rate, which occurs for $R_{\Omega}=-0.10$. This corresponds to a value of $\beta_{M d}=2 \times 10^{-5}$ or $\dot{M} / \dot{M}_{0}=3.3 \times 10^{-3}$ at $R e=7 \times 10^{5}$. Extrapolating towards astrophysical Reynolds numbers by using the formula of Hersant et al. (2005, see Eq. (6)), we get a value of $\dot{M} / \dot{M}_{0}=10^{-3}$ that is consistent with values observed in disks around T Tauri stars.

\section{Discussion}

The measured accretion rate for all of our quasi-Keplerian flows $\left(R_{\Omega} \leq-1\right)$ is approximately $14 \%$ of the maximum accretion rate, which occurs at $R_{\Omega}=-0.10$, resulting in $\beta_{\mathrm{Md}}=2 \times 10^{-5}$. This is close to the value computed by Richard and Zahn $\beta_{\mathrm{RZ}}=10^{-5}$ and to the value of Dubrulle et al. (2005) $\beta_{\mathrm{D}}=8 \times 10^{-6}$. This is somewhat surprising given that quasi-Keplerian flow profiles are linearly stable while the maximum accretion rate occurs when the cylinders counter-rotate, which produces destabilizing shear that dominates the dynamics. This result is in stark contrast to the results presented by Ji et al. (2006) and Schartman et al. (2012), where $\beta_{\mathrm{P}}$ was measured to be an order of magnitude smaller, specifically $\beta_{\mathrm{P}}=7 \times 10^{-7}$. Thus, they concluded that hydrodynamics alone cannot efficiently transport angular momentum in quasi-Keplerian flows.

Schartman et al. (2012) state that these incompatible results are caused by differing interpretations of finite-size effects, namely Ekman circulation produced by the axial boundaries. In the Princeton experiments (Ji et al. 2006; Schartman et al. 2009; Burin et al. 2010; Schartman et al. 2012) the aspect ratio is small $(\Gamma \approx 2)$ and therefore finite-size effects would likely dominate. To minimize Ekman circulation, their axial boundaries are split into pairs of rings that can independently rotate with respect to the cylinders. This method has been shown to reduce the effects of Ekman circulation in the bulk of the fluid (Schartman et al. 2009). Furthermore, when Ekman circulation is not minimized their measured values of $\beta$ for quasi-Keplerian flows are approximately half of the typical values presented here. Thus, Schartman et al. (2012) argue that our larger values of $\beta$ are the result of Ekman circulation that is present in other experiments, but is minimized in their apparatus.

We argue, on the other hand, that while finite-size effects are certainly present in our experiments, we believe that they do not dominate our measured torques. Before presenting these arguments, we would like to preface the discussion with a summary of past studies that have examined the role of finite-size effects on angular momentum transport in rotating shear flows. The work of Lesur \& Longaretti (2005) used numerical simulations of rotating plane-Couette flow up to $R e \sim 10^{4}$ in the shearing-sheet limit to conclude that Keplerian-like flows in Taylor-Couette systems are strongly affected by Ekman circulation. The aforementioned experiments by the Princeton group (Ji et al. 2006) experimentally observed that angular momentum transport in their apparatus was only significant when they did not actively minimize Ekman circulation by varying the rotation rate of their axial end rings.

To further address this issue from a numerical standpoint, Avila (2012) performed direct numerical simulations of our experimental geometry as well as the Princeton apparatus (Ji et al. 2006; Schartman et al. 2009; Burin et al. 2010; Schartman et al. 2012). Avila found that the axial boundaries drive secondary flows that enhance the global angular momentum transport. The measured torques in those simulations were equal to the laminar value near $R e=0$ and increased (approximately linearly) to be twice the laminar value in both the Maryland and Princeton geometry near his maximum $R e \approx 5000$. While it was clear that finite-size effects played a dominant role for $R e<5000$, Avila concluded by stating that the universal scaling of the torque with Reynolds number observed by Paoletti \& Lathrop (2011) indicates that shear mechanisms might overwhelm finite-size effects at the values of $R e$ explored here.

The role of finite-size effects at the values of $R e$ that we have explored remains an open question. In order to quantitatively determine their effects at $R e \sim 10^{6}$ a series of future studies will be required, as discussed at the end of this section. We now turn to our arguments as to why finite-size effects may not dominate the flows that we have examined, with the caveat that we cannot rule out the possibility that secondary flows produced by end-wall effects are one of the different mechanisms that may 
perturb our quasi-Keplerian flows into a turbulent state (see e.g. Mukhopadhyay et al. 2005; Mukhopadhyay \& Saha 2011, and references therein).

The normalized torque $G / G_{0}$ is approximately constant for each value of $R_{\Omega}$ characterized by the present experiments. This indicates that the amplitude of the torque varies with $R_{\Omega}$, however the scaling with $R e$ is unchanged (see Fig. 5). Paoletti \& Lathrop (2011) observed that their torque scaling for Rayleighstable and unstable flows agreed with the Prandtl-von Kármán description of Lathrop et al. (1992a); Lewis \& Swinney (1999). The Twente data, initially presented by van Gils et al. (2011b), was well-described by the theoretical predictions of Eckhardt et al. (2007b) for all rotation numbers explored. If Ekman circulation significantly contributed to the torque for Rayleigh-stable flows, as argued by Schartman et al. (2012), then one would expect systematic disparities between these theoretical descriptions and the measured torques. As shown in Fig. 5, though, the torque scaling agrees well with the observations of Lewis \& Swinney (1999) for the case $\Omega_{2}=0$ for all values of $R_{\Omega}$. Avila (2012) also argued that this universal scaling with $R e$ indicates that shear turbulence dominates at the Reynolds numbers that we have explored, although this does not prove that secondary flows did not have a role in initiating the shear turbulence.

The torques in the Maryland and Twente experiments are measured over only $56 \%$ of the axial length of the flow centered at the mid-height of the apparatus. This design has been intentionally implemented such that the torque measurements are unaffected by flows within 2.58 radial gap widths of either axial boundary where Ekman circulation is strongest. Wendt's experiments (Wendt 1933) had a free upper surface, which is therefore devoid of Ekman circulation. Even though Taylor's measurements (Taylor 1936) were affected by Ekman circulation for $\Gamma>100$, his measurements of the torque for Rayleigh-stable flows that are expected to be most affected by Ekman circulation agree with our measurements of counter-rotating flows for the same value of $R_{\Omega}$, where Ekman circulation is dwarfed by the dominant shear.

One of course wonders on whether this Ekman circulation caused by the co-rotating upper and lower plates affect the flow patterns. For laminar flow e.g. for purely outer cylinder rotation this clearly is the case (Coles \& van Atta 1966; Hollerbach \& Fournier 2004). However, we argue that the strong turbulence destroys such an axial dependence of the flow field. To check this assumption, we have measured the full turbulent velocity profile with laser doppler anemometry (LDA; van Gils et al. 2012). The result is shown in Fig. 7. Evidently, over the length of the central section of the inner cylinder where we measure the torque there is no visible $z$-dependence of the mean velocity; the plate boundary layers only produce an axial dependence far above the domain of the central section of the inner cylinder.

The Princeton experiments ( $\mathrm{Ji}$ et al. 2006; Schartman et al. 2009; Burin et al. 2010; Schartman et al. 2012) measure local velocity correlations to determine the global angular momentum $f l u x$, whereas the Maryland and Twente experiments directly measure the global angular momentum flux using precision calibrated force-arm measurements. The distinction between the measured quantities has made direct comparisons of the experiments difficult. To bridge this gap, Burin et al. (2010) recently used their local velocity measurements to determine the corresponding torque $G$. To compare to previous studies with $\Omega_{2}=0$ (Lathrop et al. 1992b,a; Lewis \& Swinney 1999) Burin et al. (2010) fit the scaling of $c_{\mathrm{f}}^{-1 / 2}$ to $\log _{10}\left(R e * c_{\mathrm{f}}\right)$, where $c_{\mathrm{f}} \equiv G / R e^{2}$, and determined a slope of $1.40 \pm 0.16$. The authors claim that this value agrees with the measured value of 1.52 reported by

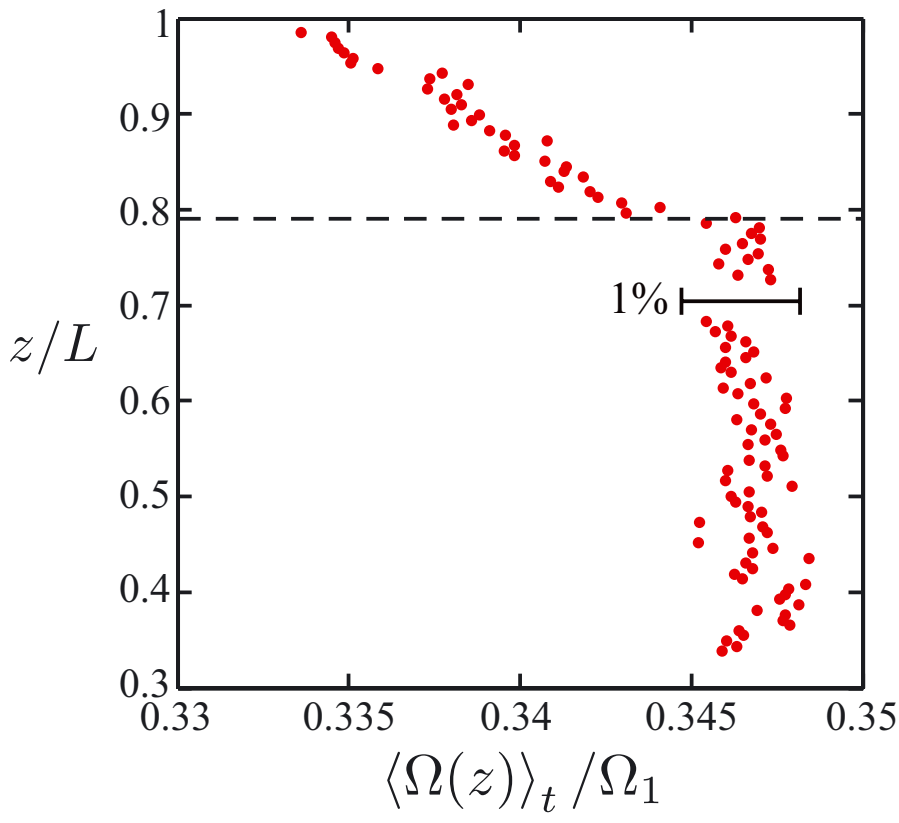

Fig. 7. Finite-size effects may be characterized by axial profiles of the angular velocity $\Omega$ measured at the mid-gap $(r=(a+b) / 2)$ in the Twente experiment. In this example, the cylinder Reynolds numbers are $R e_{1}=$ $1.0 \times 10^{6}$ and $R e_{2}=0$ (outer cylinder stationary). The global torque measurements are taken for $0.22<z / L<0.78$ with the top boundary of these measurements indicated by the dashed line at $z / L=0.78$. The resolution of the laser-doppler anemometry prohibits measurements of the angular velocity in the boundary layer near $z / L=1$ where $\Omega=$ $\Omega_{2}=0$, in this case.

Lathrop et al. (1992b,a) and 1.56 measured in Lewis \& Swinney (1999). However, the appropriate Prandtl-von Kármán scaling predicts the following relationship (Lathrop et al. 1992b,a; Lewis \& Swinney 1999)

$\frac{1}{\sqrt{c_{\mathrm{f}}}}=N \log _{10}\left(\operatorname{Re} \sqrt{c_{\mathrm{f}}}\right)+M$,

in contrast to the scaling shown in Fig. 5 of Burin et al. (2010). Furthermore, the fit parameters $N$ and $M$ are predicted to depend upon the radius ratio in the following way

$$
\begin{aligned}
& N=\left[\left(1-\eta^{2}\right) \ln 10\right] /(\eta \kappa \sqrt{2 \pi}), \\
& M=\frac{N}{\ln 10}\left[\ln \left\{\left(\frac{1-\eta}{1+\eta}\right) \frac{1}{y_{0}^{+} \sqrt{2 \pi}}\right\}+\kappa y_{0}^{+}\right],
\end{aligned}
$$

where $\kappa=0.40$ is the von Kármán constant and $y_{0}^{+}=5$. The radius ratio $\eta=0.35$ in the Princeton experiments, therefore the Prandtl-von Kármán skin friction law predicts $N=5.76$ and $M=-3.15$. These values do not agree with our analysis of the data in Burin et al. (2010) where $N \approx 2$ and $M \approx-0.4$. In fact, the measured values of $G$ determined by the local velocity fluctuations are on average five times larger than those predicted by the Prandtl-von Kármán skin friction law that has been verified using direct torque measurements (Lathrop et al. 1992b,a; Lewis \& Swinney 1999; Paoletti \& Lathrop 2011). Therefore, we argue that the local velocity fluctuation measurements used in the Princeton experiments ( $\mathrm{Ji}$ et al. 2006; Schartman et al. 2009; Burin et al. 2010; Schartman et al. 2012) face severe experimental challenges. In fact, Huisman et al. (2012) showed that the local angular momentum transport undergoes fluctuations that are two orders of magnitude larger than the mean 
value. However, once the local angular momentum transport is averaged long enough and over a large enough region, Huisman et al. (2012) achieved perfect agreement between the local and the global measurements.

Clearly the contrasting conclusions of the Princeton experiments (Ji et al. 2006; Schartman et al. 2009; Burin et al. 2010; Schartman et al. 2012) and those presented here warrant further experimental and theoretical investigation, as suggested by Balbus (2011). In particular the role of finite-size effects must be better understood at $R e \sim 10^{6}$. It may be that the Princeton measurements of the local velocity fluctuations may not accurately determine the global angular momentum transport (Burin et al. 2010), due to lack of convergence. On the other hand, the effects of the axial boundaries on the global torque measurements presented here have not yet been characterized in detail. The Twente experiments (Huisman et al. 2012) provide both local velocities as well as the global torque, which thereby presents future opportunities to clarify these distinctions. Vice versa, direct global torque measurements in a split axial-ring apparatus, such as the Princeton device, would also be scientifically useful. New experiments that also measure the angular momentum transport as a function of aspect ratio would also be very interesting.

An explanation for the contrasting results of our measurements with those of past studies can be sought in both future experiments and numerical simulations. From an experimental point of view, an apparatus that is capable of simultaneously measuring the global angular momentum transport and controlling secondary circulation by independently varying the rotation rate of split axial-rings would be particularly fruitful. In essence, such an apparatus would combine the techniques utilized in the Twente and Maryland experiments with the apparatus of the Princeton group (Ji et al. 2006; Schartman et al. 2009; Burin et al. 2010; Schartman et al. 2012). This would remove the ambiguities that are present in interpreting data from these experiments.

Avila (2012) observed that the torque increased (approximately) linearly with $R e$ and was no more than twice the laminar value for $R e<5000$. However, our measured torques are typically two orders of magnitude greater than the laminar value and increase faster than $G \propto R e$, thus indicating a transition. Additional experiments could aid future numerical studies by performing measurements at lower Reynolds number to determine when the transition to turbulence occurs. In this way, computational studies could focus their efforts on examining values of the Reynolds number that straddle the transition point. Furthermore, experimentally characterizing the angular momentum transport below the transition will also be important in determining the torque produced by secondary flows.

In addition to overcoming these experimental challenges, efforts must be made to design laboratory systems that more closely resemble the astrophysical flows of interest. In accretion disks the radial and axial length scales are comparable (Regev \& Umurhan 2008). However, many laboratory experiments (see Table 1) are designed with large aspect ratios to minimize finite-size effects. While the results in Fig. 4 seem to capture some of the dependence on the radius ratio, systematic studies with varying $\eta$ are still required to determine the precise scaling. It also remains to be seen if our results are broadly applicable to smaller aspect ratios. The radial boundaries present in laboratory experiments produce boundary layers that affect the global angular momentum transport (Eckhardt et al. 2007b). Such boundaries may be less important in accretion flows and therefore one must account for these effects when extrapolating to astrophysically-relevant control parameters. Furthermore, recent numerical simulations by Meheut et al. (2010) have indicated that stratification may also play an important role in the dynamics of accretion disks, but such effects have not been experimentally examined in high Reynolds number Taylor Couette flow. Although these flows do not capture the full complexity of astrophysical systems, they do serve as an indication that hydrodynamic instabilities can occur in Rayleigh-stable flows. The detailed mechanism that produces the instability remains an open question for future endeavor.

\section{Conclusions}

The measurements that we present here indicate that the dimensionless torque $G$ may be described as a product of functions that separately depend upon the Reynolds number $R e$ and the rotation number $R_{\Omega}$. The rotation number $R_{\Omega}$ introduced by Dubrulle et al. (2005) collapses all of the experimental data presented here for various radius ratios, aspect ratios and Reynolds numbers (see Table 1). The effects of global rotation, measured by $G / G_{0}$, where $G_{0} \equiv G\left(\operatorname{Re}, R_{\Omega}=0\right)$, are shown to be well-described by the function $f\left(R_{\Omega}\right)$ given in Eq. (12) for the entire range of $R_{\Omega}$ explored in the present studies.

The most astrophysically relevant result is the scaling of $G / R e^{2}$, which is proportional to both the turbulent viscosity parameter $\beta$ and the accretion rate $\dot{M}$. We observe that the expected accretion rate for quasi-Keplerian flows $\left(R_{\Omega} \leq-1\right)$ is approximately $14 \%$ of the maximum attainable rate for a given Reynolds number. We therefore get a dimensionless angular transport parameter $\beta_{\mathrm{Md}}=2 \times 10^{-5}$, in agreement with Richard and Zahn but more than an order of magnitude larger than the Princeton experiments. Such angular momentum transport is able to produce dimensionless accretion rates on the order of $\dot{M} / \dot{M}_{0} \sim 10^{-3}$, which are compatible with observations in disks around T Tauri stars (Hersant et al. 2005). We have argued that our results are not easily attributed to finite-size effects, such as Ekman circulation. As such, we conclude that hydrodynamics may be able to transport angular momentum at astrophysical rates in spite of the linear stability provided by the radially-increasing angular momentum profile. This level of transport must result from turbulent means, thereby implying that quasi-Keplerian flows can be nonlinearly unstable to finite amplitude disturbances.

Acknowledgements. The Maryland group would like to thank B. Eckhardt, Michael E. Fisher, C. Kalelkar, D. Martin, Harry L. Swinney and D. S. Zimmerman and acknowledge the support of Grant No. NSF-DMR 0906109. B.D. thanks the CNRS for its continuous unconditional financial support that made this fundamental research possible. The Twente collaboration thanks G. W. Bruggert for crucial work in building the $T^{3} C$ device, S. Grossmann and S. G. Huisman for discussions, and STW, which is financially supported by NWO, for financial support of this project.

\section{References}

Abramowicz, M., Brandenburg, A., \& Lasota, J.-P. 1996, MNRAS, 281, L21 Avila, M. 2012, Phys. Rev. Lett., 108, 124501

Balbus, S. A. 2011, Nature, 470, 475

Balbus, S. A., Hawley, J. F., \& Stone, J. M. 1996, ApJ, 467, 76

Beckwith, K., Armitage, P. J., \& Simon, J. B. 2011, MNRAS, 416, 361 Brandenburg, A., Nordlund, A., Stein, R. F., \& Torkelsson, U. 1995, ApJ, 446, 741

Burin, M. J., Schartman, E., \& Ji, H. 2010, Exp. Fluids, 48, 763

Canuto, V. M., Goldman, I., \& Hubickyj, O. 1984, ApJ, 280, L55

Coles, D., \& van Atta, C. 1966, J. Fluid Mech., 25, 513

Drecker, A., Hollerbach, R., \& Rudiger, G. 1998, MNRAS, 298, 1030

Dubrulle, B. 1992, A\&A, 266, 592

Dubrulle, B., \& Hersant, F. 2002, EPJ B, 26, 379

Dubrulle, B., Dauchot, O., Daviaud, F., et al. 2005, Phys. Fluids, 17, 095103

Eckhardt, B., Grossmann, S., \& Lohse, D. 2007a, Europhys. Lett., 78, 24001

Eckhardt, B., Grossmann, S., \& Lohse, D. 2007b, J. Fluid Mech., 581, 221 
M. S. Paoletti et al.: Angular momentum transport and turbulence in Keplerian flows

Esser, A., \& Grossmann, S. 1996, Phys. Fluids, 8, 1814

Fromang, S., \& Papaloizou, J. 2007, A\&A, 476, 1113

Grossmann, S., \& Lohse, D. 2011, Phys. Fluids, 23, 045108

Hawley, J. F., Balbus, S. A., \& Winters, W. F. 1999, ApJ, 518, 394

Hersant, F., Dubrulle, B., \& Huré, J.-M. 2005, A\&A, 429, 531

Hollerbach, R., \& Fournier, A. 2004, in MHD Couette Flows: Experiments and

Models, eds. R. Rosner, G. Rüdiger, \& A. Bonanno, AIP Conf. Ser., 733, 114

Huisman, S. G., van Gils, D. P. M., Grossmann, S., Sun, C., \& Lohse, D. 2012,

Phys. Rev. Lett., 108, 024501

Ji, H., Burin, M., Schartman, E., \& Goodman, J. 2006, Nature, 444, 343

King, A. R., Pringle, J. E., \& Livio, M. 2007, MNRAS, 376, 1740

Lathrop, D. P., Fineberg, J., \& Swinney, H. L. 1992a, Phys. Rev. A, 46, 6390

Lathrop, D. P., Fineberg, J., \& Swinney, H. L. 1992b, Phys. Rev. Lett., 68, 1515

Lesur, G., \& Longaretti, P.-Y. 2005, A\&A, 444, 25

Lewis, G. S., \& Swinney, H. L. 1999, Phys. Rev. E, 59, 5457

Meheut, H., Casse, F., Varniere, P., \& Tagger, M. 2010, A\&A, 516, A31

Mukhopadhyay, B. 2008, Int. J. Mod. Phys. D, 17, 467

Mukhopadhyay, B., \& Saha, K. 2011, Res. Astron. Astrophys., 11, 163

Mukhopadhyay, B., Afshordi, N., \& Narayan, R. 2005, ApJ, 629, 383

Paoletti, M. S., \& Lathrop, D. P. 2011, Phys. Rev. Lett., 106, 024501

Pringle, J. E. 1981, ARA\&A, 19, 137

Pumir, A. 1996, Phys. Fluids, 8, 3112

Regev, O., \& Umurhan, O. M. 2008, A\&A, 481, 21
Richard, D. 2001, Ph.D. Thesis, Univ, Paris VII

Richard, D., \& Zahn, J. 1999, A\&A, 347, 734

Rozyczka, M., \& Spruit, H. C. 1993, ApJ, 417, 677

Schartman, E., Ji, H., \& Burin, M. J. 2009, Rev. Sci. Instrum., 80, 024501

Schartman, E., Ji, H., Burin, M. J., \& Goodman, J. 2012, A\&A, 543, A94

Shakura, N. I., \& Sunyaev, R. A. 1973, A\&A, 24, 337

Stone, J. M., Hawley, J. F., Gammie, C. F., \& Balbus, S. A. 1996, ApJ, 463, 656

Taylor, G. I. 1936, Roy. Soc. London Proc. A, 157, 546

Umurhan, O. M., \& Regev, O. 2004, A\&A, 427, 855

van den Berg, T. H., Doering, C. R., Lohse, D., \& Lathrop, D. P. 2003, Phys. Rev. E, 68, 036307

van den Berg, T. H., Luther, S., Lathrop, D. P., \& Lohse, D. 2005, Phys. Rev. Lett., 94, 044501

van den Berg, T. H., van Gils, D. P. M., Lathrop, D. P., \& Lohse, D. 2007, Phys. Rev. Lett., 98, 084501

van Gils, D. P. M., Bruggert, G.-W., Lathrop, D. P., Sun, C., \& Lohse, D. 2011a, Rev. Scientific Instrum., 82, 025105

van Gils, D. P. M., Huisman, S. G., Bruggert, G.-W., Sun, C., \& Lohse, D. 2011b, Phys. Rev. Lett., 106, 024502

van Gils, D. P. M., Huisman, S. G., Grossmann, S., Sun, C., \& Lohse, D. 2012 J. Fluid Mech., 706, 118149

Wendt, F. 1933, Ing. Arch., 4, 577

Zeldovich, Y. B. 1981, Roy. Soc. London Proc. A, 374, 299 